\title{
Measurements to Validate the UTD Triple Diffraction Coefficient
}

\author{
Tadahiro Negishi, Student Member, IEEE, Vittorio Picco, Student Member, IEEE, \\ Douglas Spitzer, Student Member, IEEE, Danilo Erricolo, Senior Member, IEEE \\ Giorgio Carluccio, Federico Puggelli, and Matteo Albani, Senior Member, IEEE
}

\begin{abstract}
Measurement results to validate the UTD triple diffraction coefficient are presented. The experimental setup consists of multiple metallic objects, with triangular and rectangular profiles, located inside an anechoic chamber and illuminated by a sector antenna to reproduce a spherical wavefront with a Transverse Electromagnetic (TEM) incident field. Another sector antenna is moved vertically to collect electromagnetic fields across the second order UTD Incident Shadow Boundaries and in the triple diffraction transition region. The measured and theoretical fields are compared using a free space normalization. Such comparison is also validated by calculating the mean error, the standard deviation, and root mean square error that occur between the theoretical model and the measured field. The results show excellent agreement between the theoretical third order UTD solution, employing the novel triple diffraction coefficient, and the experimental results.
\end{abstract}

Index Terms-Asymptotic diffraction theory, Diffraction, Electromagnetic measurements, Geometrical theory of diffraction, Radio propagation, Uniform theory of diffraction, Electromagnetic scattering measurements, Electromagnetic edge diffraction, Electromagnetic diffraction.

\section{INTRODUCTION}

$\mathbf{T}$ HIS article discusses the first experimental validation of the Uniform Theory of Diffraction (UTD) triple diffraction coefficient for metallic wedges originally introduced by Carluccio et al. in [1]. Preliminary results were presented in [2].

The UTD [3], [4], is an efficient method to compute electromagnetic fields in electrically large problems. The UTD provides solutions for the scattering from metallic single wedges. However, when more complex geometries are involved, it may happen that one edge is positioned so that it is illuminated by the transition field of another edge. Since the transition field is not ray-optical, the cascaded application of UTD single wedge diffraction coefficients provides wrong results [5].

The problem of multiple-wedge diffraction, when various transition regions overlap, has been extensively studied in the literature by using various approaches [6]-[9]. In the

Manuscript received date1; revised date2; accepted date3. Date of publication date4; date of current version date5

T. Negishi, V. Picco, D. Spitzer and D. Erricolo are with the Department of Electrical and Computer Engineering, University of Illinois at Chicago, Chicago, IL, 60607, USA. (email: t.negishi@gmail.com; vittorio.picco@gmail.com; dspitz2@uic.edu; derric1@uic.edu).

G. Carluccio, F. Puggelli and M. Albani are with the Department of Information Engineering, University of Siena, 53100 Siena, Italy (e-mail: giorgio.carluccio@dii. unisi.it; puggelli@unisi.it; matteo.albani@dii.unisi.it).

Color versions of one or more of the figures in this paper are available online at http://ieeexplore.ieee.org.
UTD framework, for the two dimensional case of parallel edges, in [10]-[14] the authors discuss heuristic approaches involving slope diffraction and the ad-hoc adjustments of the arguments of the Fresnel function. In detail, in [10], [11], the multiple diffracted field transitional behavior is approximated by recurring to higher order slope contributions, whereas in [12]-[14], in order to reconstruct the continuity of the total field, the authors iteratively applied UTD single and slope diffraction coefficients after heuristically modifying the UTD transition function parameters. Recently in [15] measurements results have been presented to validate a heuristic approach by the same authors [16]. However, when edges are not parallel, as occurs in real life scenarios, such heuristic approaches are not applicable since they do not exhibit the proper transitional behavior. Only for double and triple diffraction, a rigorous UTD uniform description valid for any arbitrary configuration of wedges is present in the literature.

As for UTD double diffraction, early results on the proper transitional behavior of the double diffracted field were discussed in [17]-[20]. Schneider and Luebbers [21] provided an expression for the double diffraction mechanism for two separate single wedges. In [22] the authors discussed the problem of double diffraction by two parallel wedges giving a result valid also in the near-region. In [23] the authors extended the previous solution to the case of two wedges sharing a common face. Capolino et al. [24] provided a UTD coefficient for double diffraction mechanisms by two coplanar skew edges, under oblique incidence, obtaining results for the diffracted field that are valid in the near-region. In [25], these double diffraction mechanisms were repeatedly applied to evaluate the field diffracted past multiple knife edges. Finally, Albani in [26] analyzed the double diffraction mechanism for the geometry of two skew edges in arbitrary configuration and provided results that are valid in the near-field. Additionally, two-dimensional double diffraction by impedance wedges were investigated by Herman and Volakis in [27].

Related to this article is the prior experimental validation of the UTD double diffraction coefficient [24], which was addressed in [28]-[30].

This article discusses various experiments that were designed to cause a triple diffraction mechanism in order to compare measured results with theoretically computed values of the electric field by using the formulation discussed in [1]. Sufficient details are provided to allow for independent replication of the experiments that are described.

This article is structured as follows. The experimental setup 


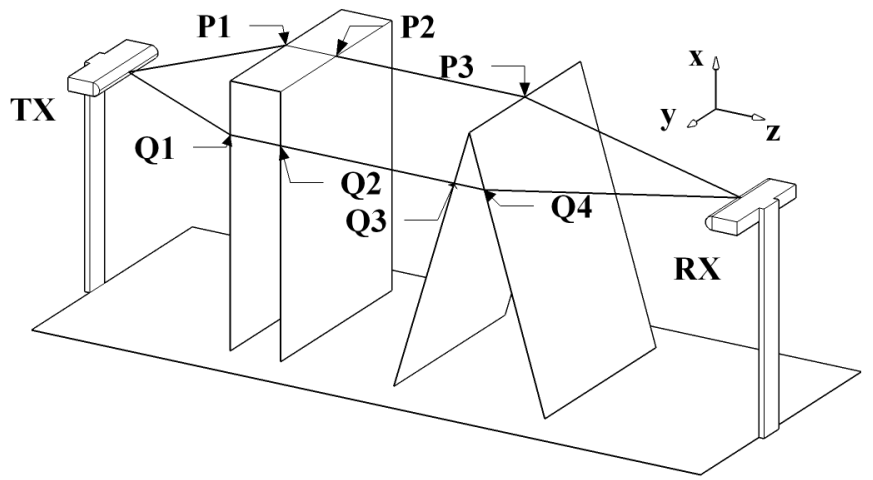

Fig. 1: 3D sketch of the experimental setup. The trajectory $\mathrm{TX} \rightarrow \mathrm{P} 1 \rightarrow \mathrm{P} 2 \rightarrow \mathrm{P} 3 \rightarrow \mathrm{RX}$ is contained in the $x z$ plane and is of interest for the experiments. Instead, the trajectory $\mathrm{TX} \rightarrow \mathrm{Q} 1 \rightarrow \mathrm{Q} 2 \rightarrow \mathrm{Q} 3 \rightarrow \mathrm{Q} 4 \rightarrow \mathrm{RX}$ is an undesired lateral wave.

is discussed in Section II and the experimental results are discussed and compared with the theoretical predictions in Section III, followed by the Conclusions.

\section{EXPERIMENTAL SETUP}

Experiments were designed to test the triple diffraction coefficient, under a spherical wavefront with a TEM field incidence, and according to the scenario of Fig. 1, which is fundamentally two-dimensional, but with a three-dimensional spreading factor. The measurements were performed in the anechoic chamber at the University of Illinois at Chicago (UIC).

Metallic obstacles of different shapes were placed between a stationary transmitting antenna (TX) and a vertically moving receiving antenna $(\mathrm{RX})$. This receiving antenna was connected to a linear positioner, which moved it by small, discrete steps along the $x$ axis. A Vector Network Analyzer (VNA, Agilent $\mathrm{N} 5222 \mathrm{~A}$ ) provided the input signal for the TX antenna and measured $S_{21}$ by sampling the electromagnetic field on the $\mathrm{RX}$ side at each step. The frequency of operation was $25 \mathrm{GHz}$ $(\lambda=11.9 \mathrm{~mm})$ for all measurements.

\section{A. Sector Antennas}

The antennas were chosen to serve two goals. One goal was to provide a spherical wavefront with a local quasi-TEM incident field at a short distance from the antennas, due to the small size of the anechoic chamber. The other goal was to suppress undesired lateral waves caused by the finite transverse size of the obstacles, as shown in Fig. 1, in order to simulate correctly a two-dimensional geometry.

These two goals can be achieved using two sets of sector antennas (Andrew Corporation, BCAH090-250 and BCAV090250), one set for each (soft and hard) polarization. These antennas possess a Half Power Beam Width (HPBW) of $90^{\circ}$ in the $x z$ plane, and a very narrow HPBW $\left(\approx 3^{\circ}\right)$ in the $y z$ plane. The antenna patterns were measured using an automated antenna positioner (Orbit/FR AL-360-1P15) with positioning precision of 0.05 degrees. Fig. 2 shows the pattern of one

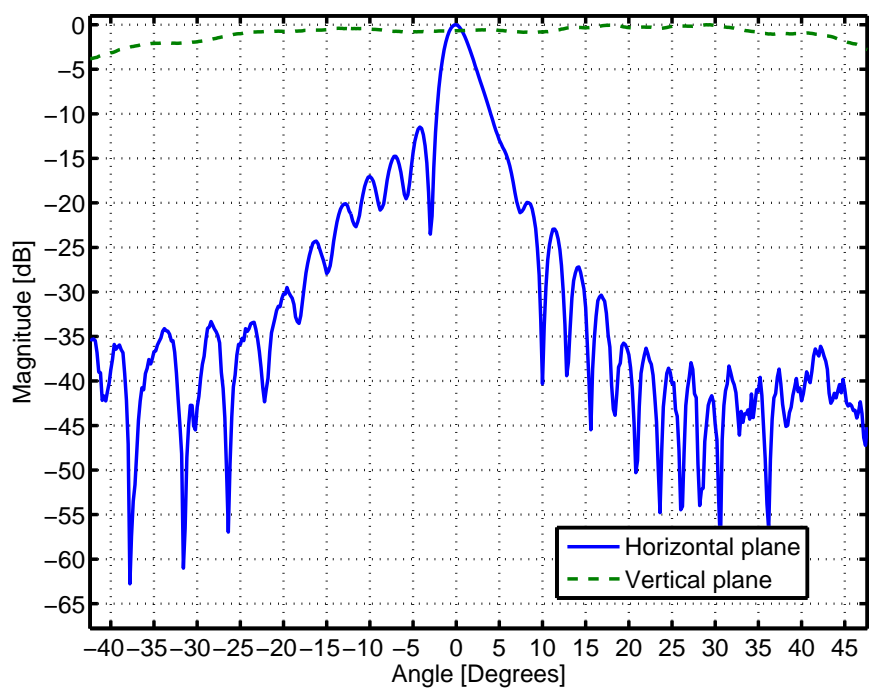

Fig. 2: Far-field radiation pattern of the sector antenna in two orthogonal planes.

of the soft-polarized antennas used in the experiments. In addition, the antennas can generate a local TEM wave at a distance of less than $1 \mathrm{~m}$ from their mouth. These two features allow to generate an incident electric field which has spherical phase fronts and uniform amplitude in the vertical $(x z)$ plane, while strongly attenuating lateral waves contributions in the horizontal $(y z)$ plane, as it is desired.

\section{B. Diffracting Objects}

In order to create three wedges, two types of metallic obstacles were used, as shown in Fig. 3. The obstacles of the first type are steel objects of triangular lateral profile, $122 \mathrm{~cm}(\approx 102 \lambda)$ wide and $85 \mathrm{~cm}(\approx 71 \lambda)$ tall. The obstacles of the second type are copper parallelepipeds of rectangular transverse profile, $20.3 \mathrm{~cm}(\approx 17 \lambda)$ deep, $76 \mathrm{~cm}(\approx 63 \lambda)$ wide and $102 \mathrm{~cm}(\approx 85 \lambda)$ tall. For both types of obstacles, the thickness of the metal was approximately $1 \mathrm{~mm}(\approx 0.1 \lambda)$. When used in combination with the chosen antennas, these objects are sufficiently wide to neglect undesired lateral ray contributions; the geometrical configurations have been chosen to guarantee an attenuation of at least $60 \mathrm{~dB}$ with respect to the main contribution.

\section{Triple Diffraction Measurements}

Diffracted fields from the three wedges were measured by reading the scattering parameter $S_{21}$ from the VNA. All measurements were conducted above the system dynamic range $(127 \mathrm{~dB})$ and the receiver dynamic range $(132 \mathrm{~dB})$, in order to be able to measure the field even within the deep shadow region. Electromagnetic absorbers were placed everywhere around the obstacles and the antennas, in order to minimize spurious scattering and diffraction of the electromagnetic fields. Particular care was taken in minimizing the scattering originating from the metallic RX antenna positioner and from the bottom edges of the metallic obstacles.

While the TX was kept at a fixed position, the RX was moved from top to bottom by a precision linear positioner, 


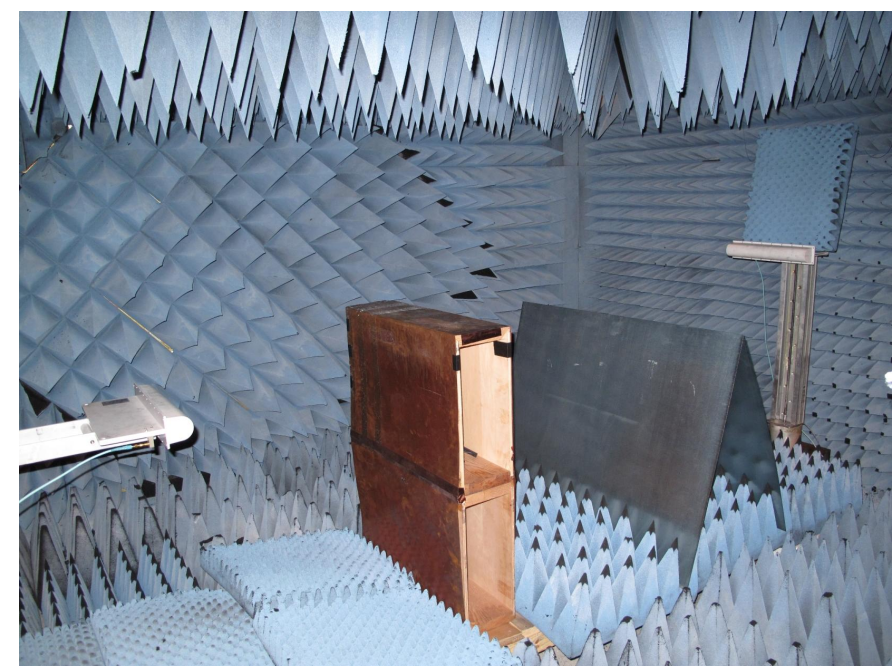

Fig. 3: Anechoic chamber at UIC. Transmitting antenna (TX) and receiving antenna $(\mathrm{RX})$ are located at each end of the room.

(controller Velmex VXM-1, stepper motor American Precision 34D-9106C, lead screw UniSlide C P40), which provided a precision of $10 \mu \mathrm{m}(\approx \lambda / 1200)$. The "zero" position corresponds to TX and RX aligned (same $x$ and $y$ position). Positive positions correspond to the RX being higher than the TX, and conversely for negative positions.

In order to obtain meaningful data, the actual positions of antennas and obstacles in the anechoic chamber must correspond as closely as possible to the one modeled in the analytic formulas. Therefore, the metallic obstacles were precisely positioned using a laser level (Craftsman Laser Trac), to make sure of the relative alignment of the obstacles with respect to both the other obstacles and the antennas. In addition, all distances were measured using a laser distance meter (Leica Disto Classic 5a), which guarantees a measurement error within $1.5 \mathrm{~mm}(\approx \lambda / 8)$.

\section{Four Case Studies}

Four case studies have been tested and are described in this article. For each case, the different geometries are created by arranging the two types of obstacles in different combinations and heights.

Case 1: Three non-aligned triangular wedges. Each wedge is placed at a different height so that each Incident Shadow Boundary (ISB) is observed separately.

Case 2: Three aligned triangular wedges. The triple diffraction UTD coefficient becomes extremely important, since the ISBs are overlapped.

Case 3: A rectangular obstacle not aligned with a triangular obstacles. The 1st and 2nd wedge share a common face. The ray fields, that reach the third wedge, propagate at grazing of the common face between the 1 st and 2 nd wedge.

Case 4: A rectangular obstacle aligned with a triangular obstacle. The 1 st and 2 nd wedge share a common face. The ray fields, that reach the third wedge, propagate again at grazing of the common face between the 1st and 2nd wedge. Furthermore, all the ISBs overlap.

A schematic representation of the four cases is shown in Fig. 4. The corresponding dimensions for all four cases have been summarized in Table I.

\section{E. Useful Definitions}

The measured field is plotted in terms of the normalized field $E_{0}(x)$, which is obtained from the appropriate component of the measured field in presence of the obstacles $\mathbf{E}(x)$, divided by the magnitude of the same component of the incident field measured for the same positions in free space $\mathbf{E}_{\text {freespace }}(x)$. This normalization to the field in free space was chosen because it has the physical meaning of additional propagation loss with respect to the free space loss. Namely,

$$
E_{0}(x)=\frac{\mathbf{E}(x) \cdot \hat{\mathbf{n}}}{\left|\mathbf{E}_{\text {freespace }}(x) \cdot \hat{\mathbf{n}}\right|}, \hat{\mathbf{n}}= \begin{cases}\hat{\mathbf{x}} & \text { hard polarization } \\ \hat{\mathbf{y}} & \text { soft polarization }\end{cases}
$$

The field predicted using the UTD triple diffraction coefficient [1] is also normalized using Eq. (1).

Two polarizations are considered for each case: hard and soft. Hard polarization corresponds to the Neumann boundary condition, which occurs when the incident electric field is perpendicular to the diffracting edge and it is also known as vertical polarization. Soft polarization corresponds to the Dirichlet boundary condition, which occurs when the incident electric field is parallel to the diffracting edge and it is also known as horizontal polarization.

In all figures, the locations of ISBs are indicated with thick vertical black lines on the horizontal axis. In details, ISB1 represents the GO shadow boundary, where the singly diffracted field undergoes a transition; ISB2 represents the shadow boundary of the first order UTD solution, where doubly diffracted field undergoes a transition; and ISB3 represents the shadow boundary of the second order UTD solution, where triply diffracted field undergoes a transition.

Details of the implementation of the UTD triple diffraction coefficient can be found in [1]. In all computations it is assumed that fields propagate from the transmitter to the receiver, neglecting any backscattered contribution.

The agreement between the fields computed with the third order UTD solution and the measured fields was evaluated in terms of Mean Error (ME), Standard Deviation (SD) and Root Mean Square Error (RMSE). The ME is a good indicator for the overall behavior and cancels spurious contributions by averaging them out. The SD and RMSE require the computation of the square of the distance between the curve for the theoretical values and the curve for the measured values. The closer these curves are to each other, the smaller SD and RMSE are.

Accordingly, we first define the error between the measurement and the theoretical prediction as function of the receiver position along the $x$ axis. For convenience, the position along 


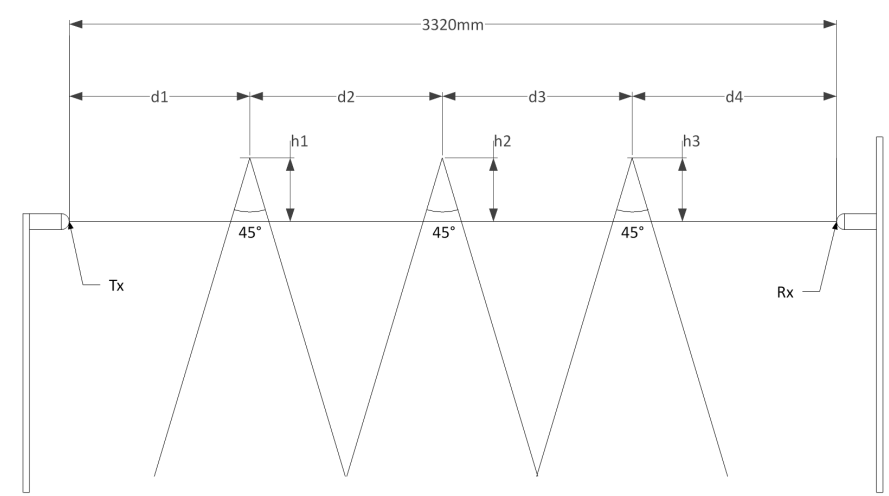

(a)

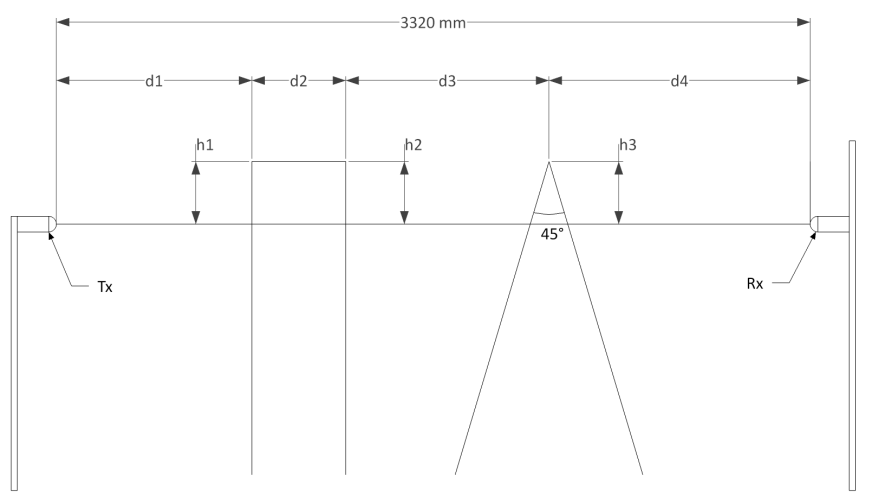

(b)

Fig. 4: Lateral view of geometries for cases 1 and 2 (a) and cases 3 and 4 (b).

TABLE I: Geometrical parameters and locations of the ISBs in millimeters and wavelengths. ISB2 and ISB3 in case 2 and case 4 overlap because of alignments.

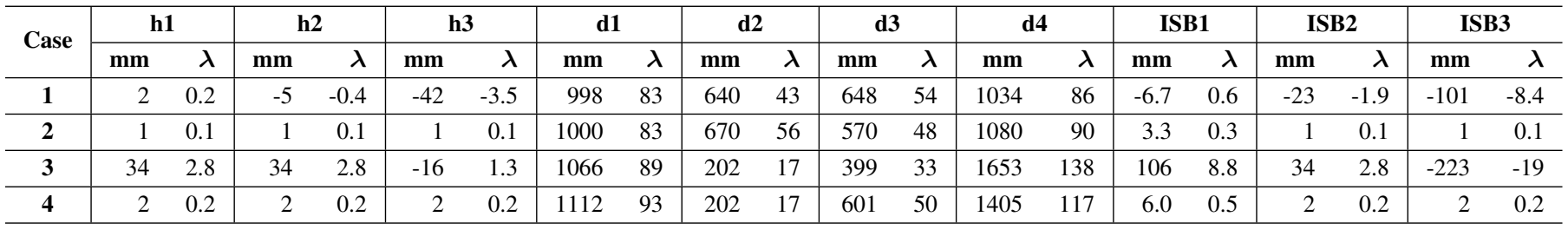

the axis is identified with the auxiliary index $p$ :

$$
\Delta(p)=E_{0}^{\text {meas }}(p)-E_{0}^{\mathrm{UTD} 3}(p) .
$$

The subscript 0 indicates that the quantities were normalized to their respective incident field in free space values, as explained in Eq. (1); all values are expressed in $\mathrm{dB}$. Then, the ME is computed as:

$$
\mathrm{ME}=\frac{1}{P} \sum_{x=1}^{P} \Delta(p),
$$

where $P$ indicates the total number of positions sampled during a linear scan.

The SD is computed as

$$
\mathrm{SD}=\left[\frac{1}{P-1} \sum_{p=1}^{P}(\Delta(p)-\mathrm{ME})^{2}\right]^{\frac{1}{2}} .
$$

The RMSE is computed as

$$
\operatorname{RMSE}=\left[\frac{1}{P} \sum_{p=1}^{P}|\Delta(p)|^{2}\right]^{\frac{1}{2}} .
$$

The statistics defined in Eqs. (3)-(5) were calculated for all cases, and are summarized in Table II, which shows excellent agreement between measurements and theoretical prediction. The ME is smaller than $1 \mathrm{~dB}$ in all cases except one, showing that the asymptotic behavior of the measurements follows with great precision the theoretical expectation. The SD is larger than $1 \mathrm{~dB}$ in only two cases, and the RMSE is always smaller than $2 \mathrm{~dB}$, and smaller than $1 \mathrm{~dB}$ in half the cases tested.
TABLE II: Mean Error, Standard Deviation, and Root Mean Square Error between UTD predicted field and measured field for each case.

\begin{tabular}{c|cccc}
\hline Case & Polarization & ME [dB] & SD [dB] & RMSE [dB] \\
\hline \multirow{2}{*}{1} & Soft & -0.11 & 1.10 & 1.10 \\
\cline { 2 - 5 } & Hard & 1.08 & 0.44 & 1.16 \\
\hline \multirow{2}{*}{2} & Soft & 0.16 & 1.69 & 1.70 \\
\cline { 2 - 5 } & Hard & 0.35 & 0.60 & 0.69 \\
\hline \multirow{2}{*}{3} & Soft & -0.76 & 0.86 & 1.15 \\
\cline { 2 - 5 } & Hard & 0.64 & 0.27 & 0.70 \\
\hline \multirow{2}{*}{4} & Soft & -0.07 & 0.60 & 0.61 \\
\cline { 2 - 5 } & Hard & 0.86 & 0.17 & 0.88 \\
\hline
\end{tabular}

\section{DiscusSiOn OF THE RESUlTS}

In Fig. 5 and in Fig. 6 the magnitude of the fields are shown. Thick black solid lines indicate measured fields, thick red dashed lines show the third order UTD fields computed by using the novel UTD triple diffraction coefficient [1]. Furthermore, thin blue dash lines report the field obtained by describing the double and triple diffracted rays with the simple cascading of standard (singly) diffraction UTD coefficient, as it is frequently done in available codes. To emphasize the instability of the latter approach to the geometrical parameters, the thin magenta dash-dot lines represent the same formulation by cascading standard UTD solution when a negligible shift of the 2nd edge in the $x$ direction of $+10^{-9} \mathrm{~m}\left(\approx 10^{-7} \lambda\right)$ slightly breaks the alignment of Case 2 and Case 4 .

For all four cases, the measurements follow the theoretical expectation with very good accuracy. The measurements 


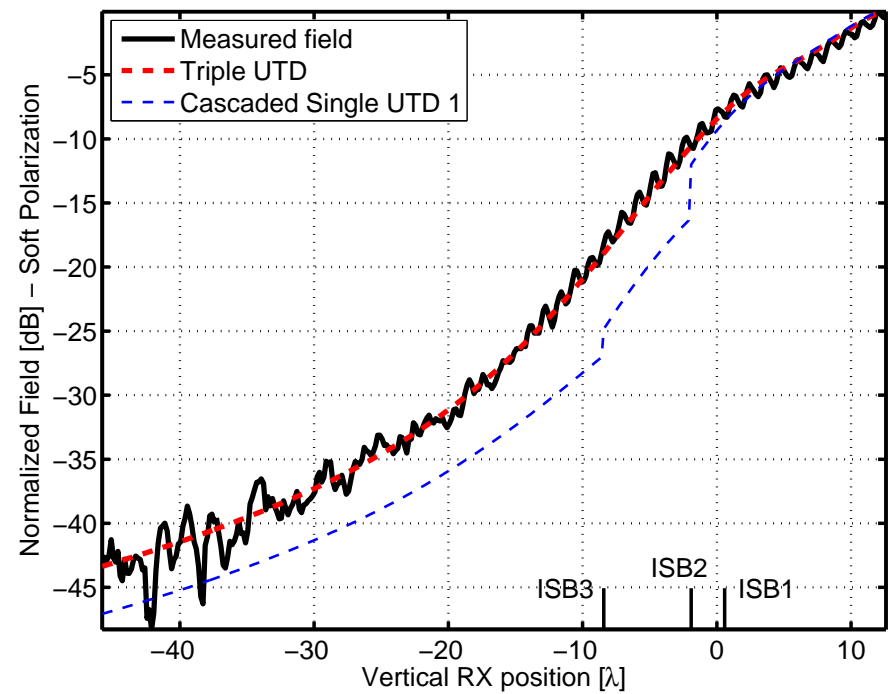

(a)

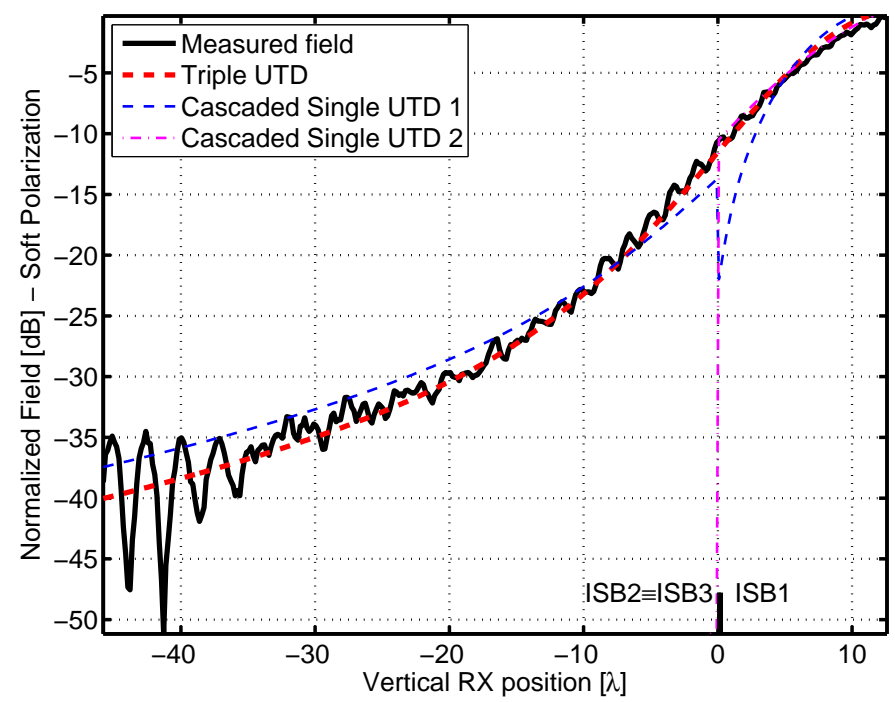

(c)

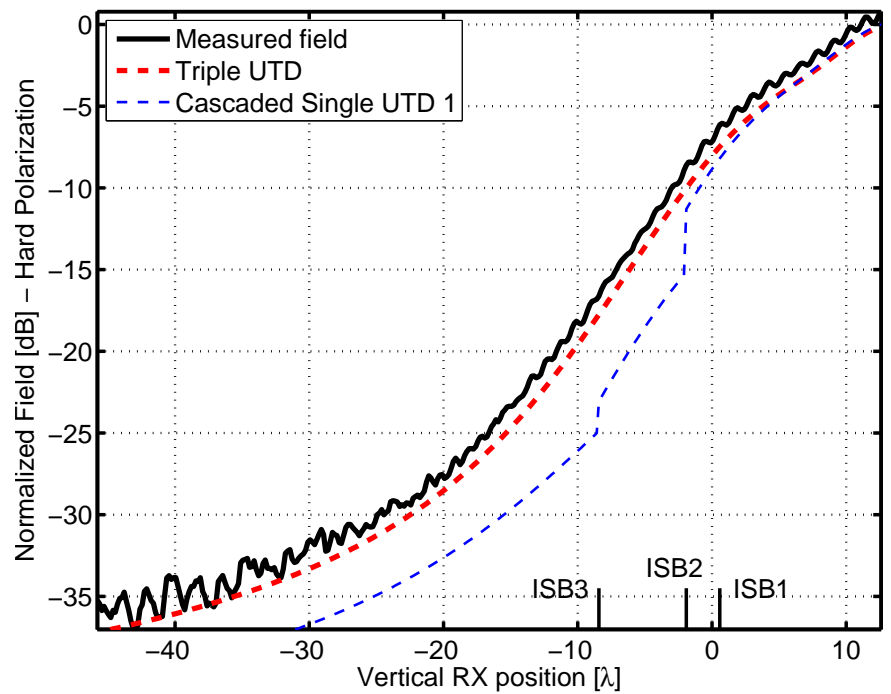

(b)

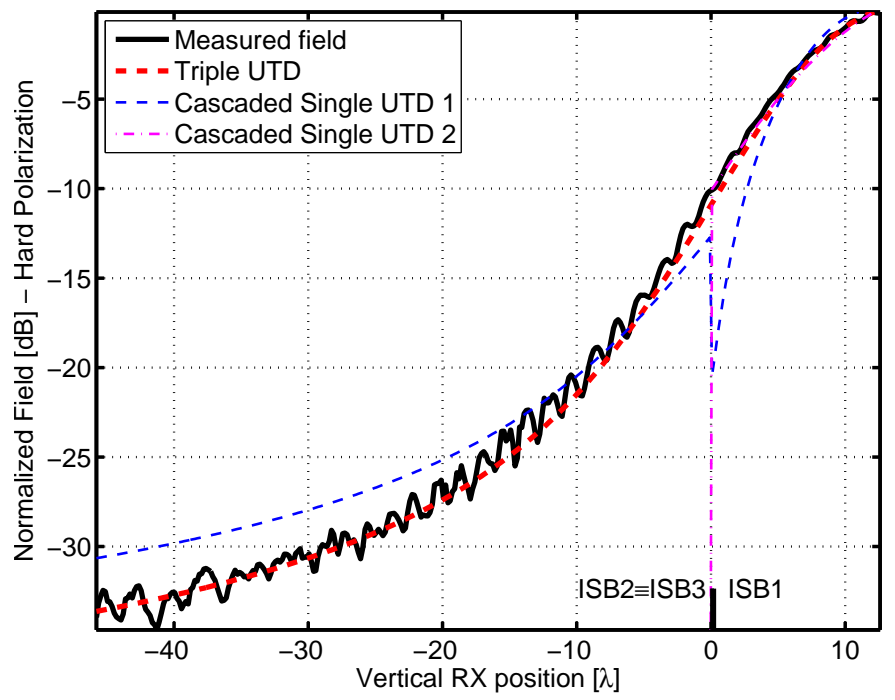

(d)

Fig. 5: Normalized measured and calculated electric field, for the geometries involving obstacles with triangular cross-section. Case 1, (a) soft polarization and (b) hard polarization. Case 2, (c) soft polarization and (d) hard polarization; notice that the magenta dash-dot curve, corresponding to the cascaded first order UTD 2, exhibits a jump discontinuity, dropping to a very low level field, which is out of scale, when the receiver is at the ISB3.

confirm the theoretical prediction in all regions, including the deep shadow on the left of the ISB3. The hard polarization of Case 1, Case 3, and Case 4 has a constant shift from the theoretical expectation. Authors believe this is due to errors in the normalization to the free space measurement, which affected the hard polarization more than the soft one.

Small ripples are observed for all cases, which are symptoms of multiple ray contributions caused by interference. These may be attributed to three contributions. The first contribution is due to reflections coming from an imperfect anechoic environment. Since the antenna pattern is very wide in the vertical plane, a large signal is sent towards both the floor and the ceiling. While the floor contribution is shielded by the obstacles themselves, the signal impinging on the ceiling is only attenuated by the EM absorbers, which allow some spurious reflection to reach the RX. The second contribution is due to interactions which occurred between the obstacle closest to the RX and the linear RX positioner itself. Relatively small ME and large SD in the soft polarization cases indicate larger error due to multiple ray contributions because (i) diffraction is weaker for soft polarization than for hard polarization so that the rays are comparable to the main contribution; and (ii) the exposed area of the metal frame of the receiver is quite large and cannot be covered by EM absorbers. The third contribution is due to lateral waves originating from the truncated sides of the obstacles, which are greatly attenuated by the antennas radiation pattern, but cannot be completely eliminated. The relative significance of these 


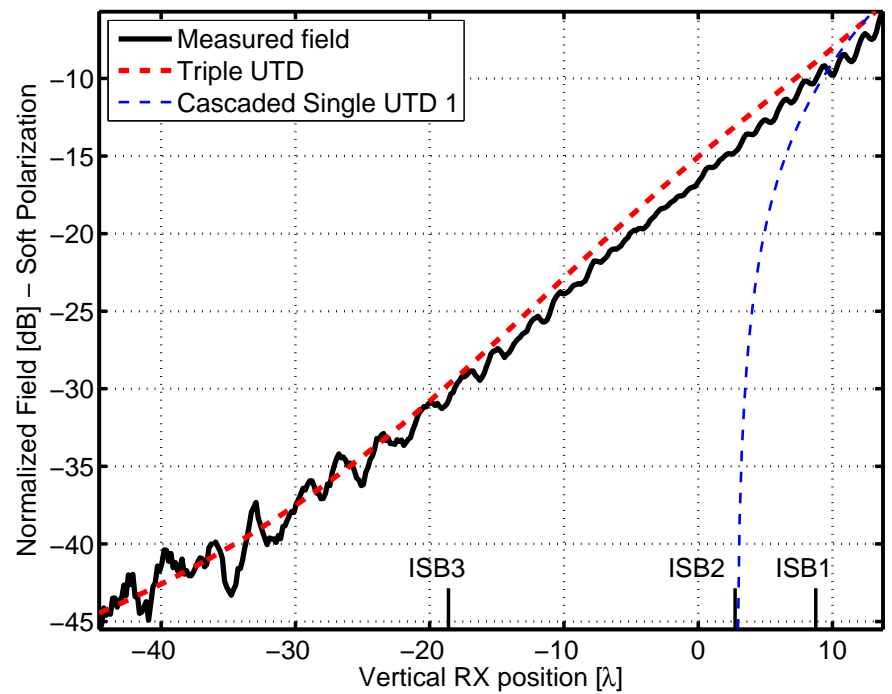

(a)

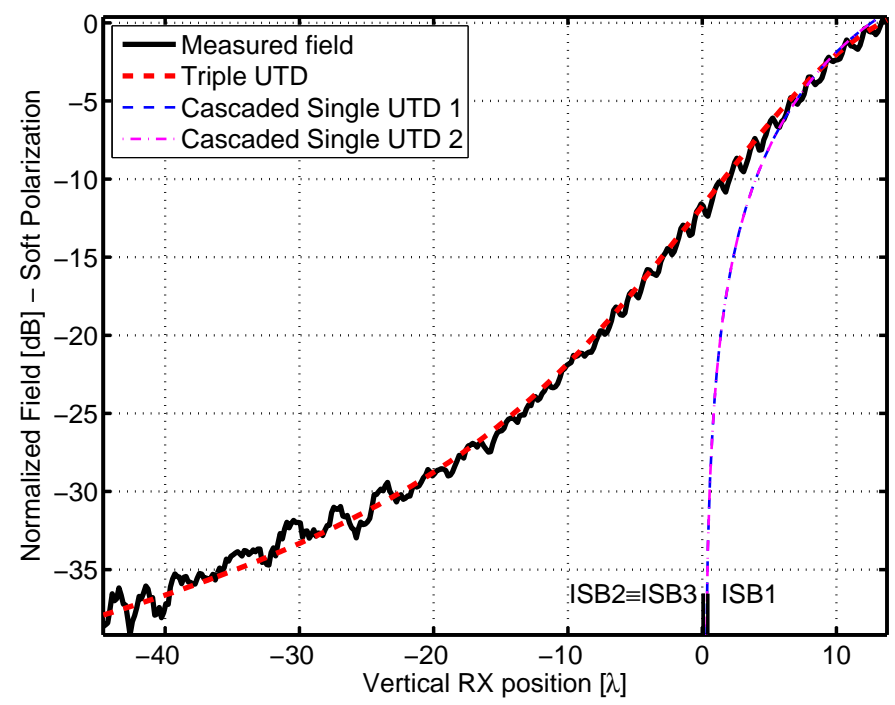

(c)

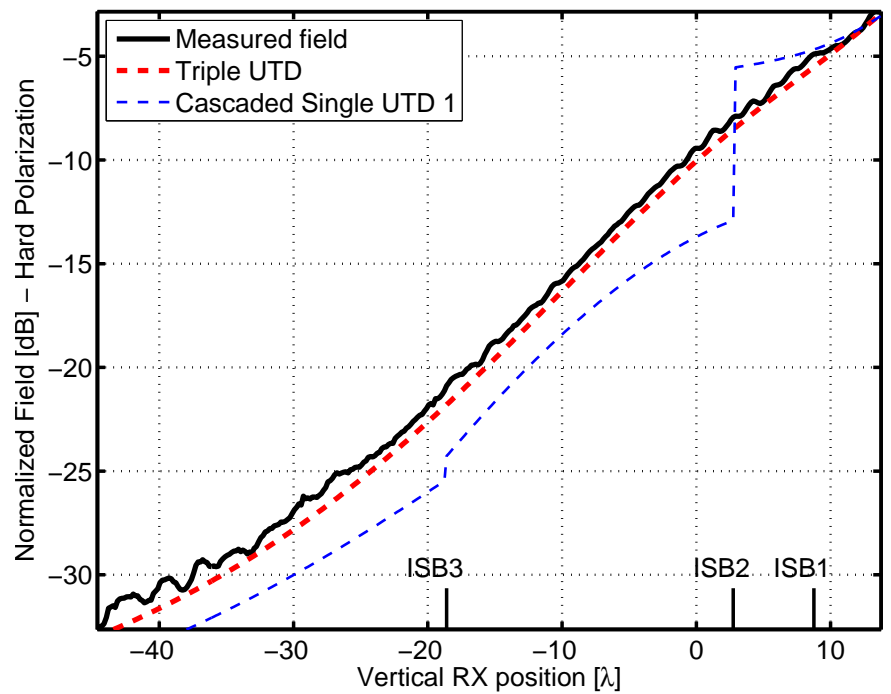

(b)

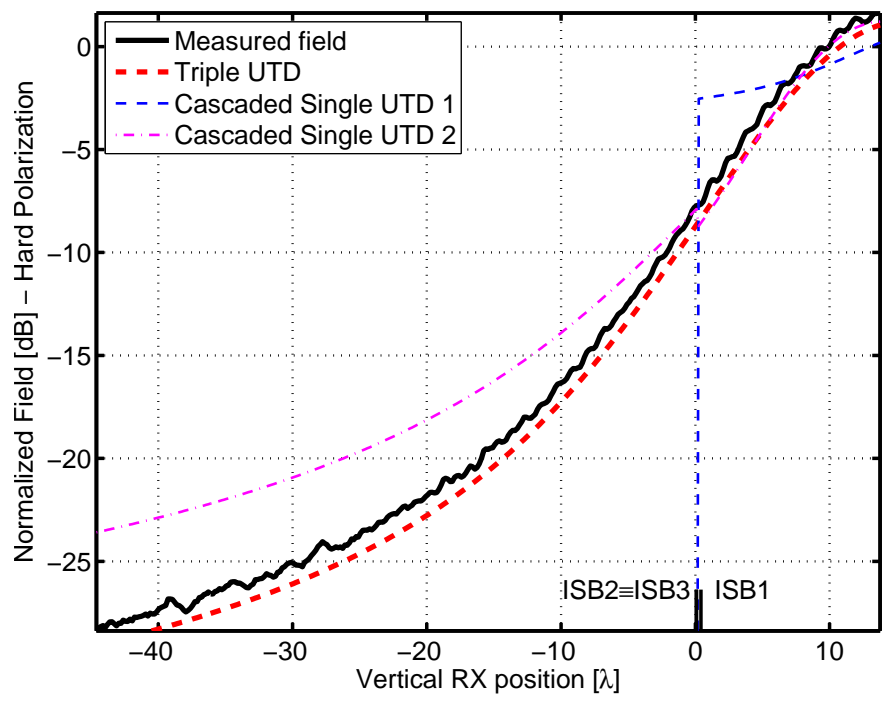

(d)

Fig. 6: Normalized measured and calculated electric field, for the geometries involving obstacles with rectangular and triangular cross-sections. Case 3, (a) soft polarization and (b) hard polarization. Case 4, (c) soft polarization and (d) hard polarization. In Fig. 6c the two cascaded first order UTD results overlap.

contributions increases with smaller measured fields, therefore we expect it to become significant only in the deeply shadowed region.

The comparison between the measurements and the cascaded first order UTD prediction shows, as expected, that only the proper UTD triple diffraction coefficient can correctly describe all the interactions among obstacles and the transitions between different shadow regions. In particular, since the direct contribution (geometrical optics) between TX and RX was blocked by the first obstacle, the cascaded first order UTD theory shows two sharp transitions at the locations of the second and third shadow boundaries. This happens for all cases and provides further motivations for the use of the UTD triple diffraction coefficient.

When all obstacles are aligned such as in Case 2 and Case
4, three ISBs are also aligned, that is, the received field crosses three incident shadow boundaries at once. While in a simulator it is trivial to set this condition, in reality it is practically impossible to guarantee perfect alignment. Very small errors in the actual alignment of the objects can lead to very large differences in the field computed by cascading the UTD single diffraction coefficients.

Fig. 5c and 5d show two different curves for the cascaded first order UTD. The first curve, Cascaded First order UTD 1 , corresponds to an exactly aligned geometry. The second curve, Cascaded First order UTD 2, corresponds to the case where the middle obstacle has not been aligned properly, and it sits only $10^{-9} \mathrm{~m}\left(\approx 10^{-7} \lambda\right)$ above the other two obstacles. In this case, the first curve indicates the field dropping before ISB3, then raising up again after the ISB3. On the other hand, 
because the second obstacle slightly changes the location of the second shadow boundary, the Fresnel transition function changes its behavior. As a result, the second curve indicates a rapid drop of the fields, which approaches a very low level in the $\mathrm{dB}$ scale of Fig. $5 \mathrm{c}$ and $5 \mathrm{~d}$. As a result, another argument in favor of the use of the UTD triple diffraction coefficient is to avoid the extreme sensitivity shown by the cascaded UTD single diffraction coefficients, for a movement as small as approximately $10^{-7} \lambda$. The reason of this not proper behavior of the multiple diffracted field by cascading UTD single wedge diffraction coefficient has been slightly discussed above in the paper. The reader, who is interested in this aspect, can find more details in [1], [5], [17]-[26].

Case 3 and Case 4 further validate the UTD triple diffraction coefficient. In such cases the prediction by using the cascaded UTD single diffraction coefficients is clearly wrong for soft polarization because, since the second edge is illuminated at grazing incidence by the field diffracted by the first edge which present a null at grazing, the double diffracted field by the first and the second edge and the triple diffracted field are zero. This also happens for the double diffracted field by the first and the third edge in Case 4, where all the edges are aligned. Also in Case 4 we reported the curve relevant to the scenario where the second edge was slightly moved of $\approx 10^{-7} \lambda$.

\section{CONCLUSION}

Experimental results at the frequency of $25 \mathrm{GHz}$ have been presented to validate the theoretical formulation of the UTD triple diffraction coefficient presented in [1]. Four case studies show a very good agreement between theory and measurements, as proven by Mean Error, Standard Deviation and Root Mean Square Error.

The differences between theory and experiments are believed to be due to spurious ray contributions, in particular to multiple reflections between the surface of the third wedge and the metallic frame of the receiver.

A comparison between the measurements and the values predicted by cascading UTD single diffraction coefficients has also been presented and provides both validation to and motivation for the use of the UTD triple diffraction coefficient when diffraction involves three edges in their transition zones.

\section{ACKNOWLEDGMENT}

This research was supported by AFRL through grant FA9550-12-1-0174 and the UIC Chancellor Undergraduate Research Award. The authors would like to thank Mr. ShaoLun Chien for measurements. The authors are also thankful to the anonymous Reviewers for their comments that helped improve the quality of this manuscript.

\section{REFERENCES}

[1] G. Carluccio, F. Puggelli, and M. Albani, "A UTD Triple Diffraction Coefficient for Straight Wedges in Arbitrary Configuration," IEEE Trans. Antennas Propag., vol. 60, no. 12, pp. 5809-5817, Dec. 2012.

[2] V. Picco, T. Negishi, D. Spitzer, G. Carluccio D. Erricolo, F. Puggelli, and M. Albani, "Experimental Validation of the UTD Third Order Diffraction Coefficient," in IEEE Antennas and Propagation Society International Symposium/USNC-URSI National Radio Science Meeting, Orlando, FL, USA, July 9-13, 2013.
[3] R. G. Kouyoumjian and P. H. Pathak, "A uniform geometrical theory of diffraction for an edge in a perfectly conducting surface," Proc. IEEE, vol. 62, no. 11, pp. 1448-1461, Nov. 1974.

[4] Prabhakar H. Pathak, Antenna Handbook, chapter Techniques for HighFrequency Problems, Van Nostrand, New York, 1993.

[5] S.-W. Lee, Y. Rahmat-Samii, and R. C. Menendez, "GTD, ray field and comments on two papers," IEEE Trans. Antennas Propag., vol. 26, no. 2, pp. 352-354, Mar. 1978 .

[6] S. W. Lee, "Path integrals for solving some electromagnetic edge diffraction problems," J. Math. Phys., vol. 19, no. 6, pp. 1414-1422, June 1978.

[7] L. E. Vogler, "An attenuation function for multiple knife-edge diffraction," Radio Sci., vol. 17, no. 6, pp. 1541-1546, Nov.-Dec. 1982.

[8] H. H. Xia and H. L. Bertoni, "Diffraction of cylindrical and plane waves by an array of absorbing half-screens," IEEE Trans. Antennas Propag., vol. 40, no. 2, pp. 170-177, Feb. 1992.

[9] J. H. Whitteker, "A generalized solution for diffraction over a uniform array of absorbing half-screens," IEEE Trans. Antennas Propag., vol. 49, no. 6, pp. 934-938, June 2001

[10] P. D. Holm, "UTD-diffraction coefficients for higher order wedge diffracted fields," IEEE Trans. Antennas Propag., vol. 44, no. 6, pp. 879-888, June 1996.

[11] P. D. Holm, "Calculation of higher order diffracted fields for multipleedge transition zone diffraction," IEEE Trans. Antennas Propag., vol. 52, no. 5, pp. 1350-1355, May 2004.

[12] J. Bach Andersen, "UTD multiple-edge transition zone diffraction," IEEE Trans. Antennas Propag., vol. 45, no. 7, pp. 1093-1097, July 1997.

[13] C. Tzaras and S. R. Saunders, "An improved heuristic UTD solution for multiple-edge transition zone diffraction," IEEE Trans. Antennas Propag., vol. 49, no. 12, pp. 1678-1682, Dec. 2001.

[14] M. B. Tabakcioglu and A. Kara, "Improvements on slope diffraction for multiple wedges," Taylor \& Francis, Electromagnetics, vol. 30, no. 3, pp. 285-296, Mar. 2010

[15] M. T. Martínez-Inglés, J. V. Rodríguez, J. M. Molina-García-Pardo, J. Pascual-García, and L. Juan-Llácer, "Comparison of a UTD-PO formulation for multiple-plateau diffraction with measurements at 62 GHz," IEEE Trans. Antennas Propag., vol. 61, no. 2, pp. 1000-1003, Feb. 2013.

[16] J. V. Rodríguez, J. M. Molina-García-Pardo, and L. Juan-Llácer, "A new solution expressed in terms of UTD coefficients for the multiple diffraction of spherical waves by a series of buildings," Radio Sci., vol. 42, no. 4, 2007.

[17] R. Tiberio and R. G. Kouyoumjian, "A uniform GTD solution for the diffraction by strips illuminated at grazing incidence," Radio Sci., vol. 14, no. 6, pp. 933-941, Nov.-Dec. 1979.

[18] R. Tiberio and R. G. Kouyoumjian, "An analysis of diffraction at edges illuminated by transition region fields," Radio Sci., vol. 17, no. 2, pp. 323-336, Mar.-Apr. 1982.

[19] R. Tiberio and R. G. Kouyoumjian, "Calculation of the high-frequency diffraction by two nearby edges illuminated at grazing incidence," IEEE Trans. Antennas Propag., vol. AP-32, no. 11, pp. 1186-1196, Nov. 1984.

[20] R. Tiberio, G. Manara, G. Pelosi, and R. G. Kouyoumjian, "Highfrequency electromagnetic scattering of plane waves from double wedges," IEEE Trans. Antennas Propag., vol. AP-37, no. 9, pp. 11721180, Sept. 1989.

[21] M. Schneider and R. J. Luebbers, "A general, uniform double wedge diffraction coefficient," IEEE Trans. Antennas Propag., vol. 39, no. 1, pp. 8-14, Jan 1991.

[22] L. P. Ivrissimtzis and R. J. Marhefka, "A note on double edge diffraction for parallel wedges," IEEE Trans. Antennas Propag., vol. 39, no. 10, pp. $1532-1537$, Oct. 1991

[23] M. Albani, F. Capolino, S. Maci, and R. Tiberio, "Diffraction at a thick screen including corrugations on the top face," IEEE Trans. Antennas Propag., vol. 45, no. 2, pp. 277-283, Feb. 1997.

[24] F. Capolino, M. Albani, S. Maci, and R. Tiberio, "Double diffraction at a pair of coplanar skew edges," IEEE Trans. Antennas Propag., vol. 45, no. 8, pp. 1219-1226, Aug. 1997.

[25] D. Erricolo and P. L. E. Uslenghi, "Comparison between ray-tracing approach and empirical models for propagation in urban environments," IEEE Trans. Antennas Propag., vol. 50, no. 5, pp.766-768, May 2002.

[26] M. Albani, "A uniform double diffraction coefficient for a pair of wedges in arbitrary configuration," IEEE Trans. Antennas Propag., vol. 53, no. 2, pp. 702-710, Feb. 2005.

[27] M. I. Herman and J. L. Volakis, "High frequency scattering by a double impedance wedge," IEEE Trans. Antennas Propag., vol. 36, no. 5, pp. 664-678, May 1988. 
[28] D. Erricolo, "Experimental validation of second order diffraction coefficients for computation of path-loss past buildings," IEEE Trans. Electromagn. Compat., vol. 44, no. 1, pp. 272-273, Feb. 2002.

[29] D. Erricolo, G. D'Elia, and P. L. E. Uslenghi, "Measurements on scaled models of urban environments and comparisons with ray-tracing propagation simulation," IEEE Trans. Antennas Propag., vol. 50, no. 5, pp. 727-735, May 2002.

[30] D. Erricolo, U. G. Crovella, and P. L. E. Uslenghi, "Time-domain analysis of measurements on scaled urban models with comparisons to ray-tracing propagation simulation," IEEE Trans. Antennas Propag., vol. 50, no. 5, pp. 736-741, May 2002.

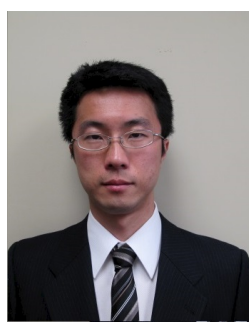

Tadahiro Negishi Tadahiro Negishi (S'12) received the B.S. in Electrical and Electronics Engineering from the Nippon Institute of Technology, Saitama, Japan, in 2007 and the M.S. degree in Electrical and Computer Engineering from the University of Illinois at Chicago, Chicago, IL, USA, in 2011. He is currently pursuing a Ph.D. degree at the University of Illinois at Chicago, working on electromagnetics related topics.

His research interests include electromagnetic wave propagations, inverse scattering and remote sensing, in particular ground penetrating radar with narrow-band signals. He has been awarded a travel grant from the National Academies to present research results at the National Radio Science Meeting in Boulder, CO in 2013 and 2014. He has co-authored 4 articles in international peerreviewed scientific journals and 17 presentations at peer-reviewed international conferences.

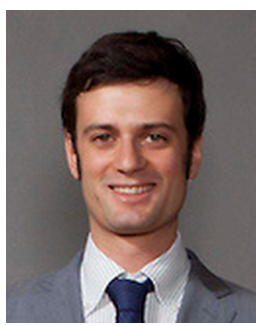

Vittorio Picco (S'09) was born in 1985 in Mondovì, Cuneo, Italy. He received the Laurea degree in electronic engineering and the M.S. (cum laude) in telecommunications engineering from the Politecnico di Torino, Italy, in 2007 and 2010, respectively. He received the M.S. in electrical and computer engineering from the University of Illinois at Chicago (UIC) in 2010. He is pursuing his Ph.D. degree in electrical and computer engineering at UIC, in the Andrew Electromagnetics Laboratory. In 2011 he was a finalist in the 2011 IEEE AP-S student design contest. He has been awarded a travel grant from the National Academies to present research results at the National Radio Science Meeting in Boulder, CO in 2012 and 2013. In 2012 and 2013 he was with Schlumberger, researching electrical and electromagnetic methods for the measurement of rock resistivity and the characterization of geological formations.

His research interests include electromagnetic propagation and scattering with applications to remote sensing and narrow-band non-destructive imaging. $\mathrm{He}$ is also interested in electrical methods for geological exploration.

Mr. Picco is a member of IEEE Eta Kappa Nu.

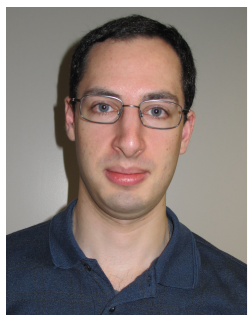

Douglas Spitzer (S'12) is currently an undergraduate senior in the department of Electrical and Computer Engineering at the University of Illinois at Chicago. Since 2012 he has been an undergraduate research assistant.

His research involves the study of electromagnetic scattering, measurement at microwave frequencies and instrumentation.

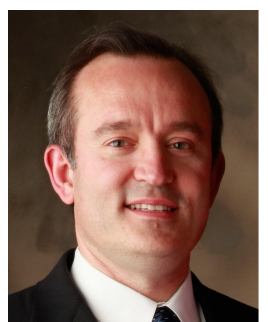

Danilo Erricolo (S'97-M'99-SM'03) received the Laurea degree of Doctor (summa cum laude) in electronics engineering from the Politecnico di Milano, Milano, Italy, in 1993 and the Ph.D. degree in electrical engineering and computer science from the University of Illinois at Chicago (UIC), Chicago, IL, USA, in 1998. He is a Professor with the Department of Electrical and Computer Engineering, UIC, where he is also the Director of the Andrew Electromagnetics Laboratory.

His research interests are primarily in the areas of electromagnetic scattering, magnetic resonance imaging, radar, wireless communications, and electromagnetic compatibility. His research activity has been supported by the Department of Defense and the National Science Foundation. He has authored or coauthored more than 180 publications in refereed journals and presentations at international conferences, and three book chapters. In 2009, he was a U.S. Air Force Summer Faculty Fellow.

Dr. Erricolo is a member of Eta Kappa $\mathrm{Nu}$ and was elected a full member of Commissions B, C and E of the U.S. National Committee of the International Union of Radio Science (USNC-URSI), a committee of the National Academies. He served for USNC-URSI Commission E as Secretary (20042005), Vice-Chair (20062008), and Chair (20092011); between 20092014 he has served as Chair of the USNC-URSI Ernest K. Smith Student Paper Competition, and he was elected Member at Large of USNC-URSI for the triennium 2012-2014. He is an Associate Editor for the IEEE Transactions on Antennas and Propagation, the IEEE Antennas and Wireless Propagation Letters, and Radio Science. He was the Vice-Chair of the Local Organizing Committee of the XXIX URSI General Assembly (Chicago, IL, USA, August $716,2008)$ and the General Chair of the 2012 IEEE Antennas and Propagation International Symposium/USNC National Radio Science Meeting (Chicago, IL, USA, July 814, 2012). He was elected as member of the Administrative Committee of the IEEE Antennas and Propagation Society for the triennium 2012-2014.

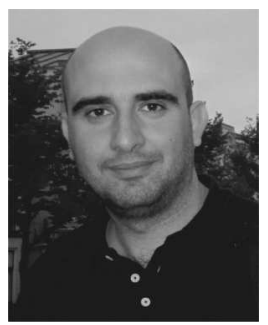

Giorgio Carluccio was born in 1979 and grew up in Ortelle, Lecce, Italy. He received the Laurea degree in telecommunications engineering and the Ph.D. degree in information engineering from the University of Siena, Siena, Italy, in 2006 and 2010, respectively.

From October 2008 to March 2009 he was an Invited Visiting Scholar with the ElectroScience Laboratory, Department of Electrical and Computer Engineering, at The Ohio State University, Columbus, Ohio, USA. From February 2010 to January 2012, he was a Postdoctoral Research Associate with the Department of Information Engineering at the University of Siena. From April 2012 to March 2013, he was a Postdoctoral Research Associate with the Department of Electronics and Telecommunication at the University of Firenze, Firenze, Italy. Since July 2013, he has been a Postdoctoral Research Associate with the Department of Information Engineering and Mathematics at the University of Siena. In 2012 and 2013 he was involved in the Research Networking Program NEWFOCUS (New Frontiers in Millimeter/Sub-Millimeter Waves Integrated Dielectric Focusing Systems) of the European Science Foundation (ESF), within a cooperation between the University of Siena and the Delft University of Technology, Department of Electrical Engineering, Mathematics and Computer Science, Delft, The Netherlands, where he was a visiting PostDoc Researcher.

His research interests are focused on asymptotic high-frequency techniques for electromagnetic scattering and propagation. Recently, he also worked on the development of algorithms for the analysis and design of dielectric lens antennas and of reflectarray antennas. 


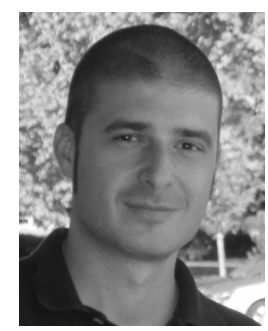

Federico Puggelli was born in Prato in 1978. He received the Laurea degree in telecommunications engineering from the University of Florence in 2007, and the Ph.D. degree in information engineering from the University of Siena in 2012.

Since 2012, he has been collaborating with the ElectroScience Laboratory, Department of Electrical and Computer Engineering, at The Ohio State University, Columbus, Ohio, USA, where he was an Invited Visiting Scholar from March to August 2012, under the supervision of Professor P. H. Pathak.

His research interests include electromagnetics and modeling of fading channels.

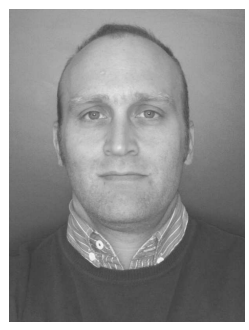

Matteo Albani received the Laurea degree in electronic engineering (1994) and the Ph.D. degree in telecommunications engineering (1999) from the University of Florence, Italy.

$\mathrm{He}$ is an Adjunct Professor in the Information Engineering and Mathematics Department, University of Siena, Italy, where he is also Director of the Applied Electromagnetics Lab.

His research interests are in the areas of highfrequency methods for electromagnetic scattering and propagation, numerical methods for array antennas, antenna analysis and design.

Dr. Albani was awarded the "Giorgio Barzilai" prize for the Best Young Scientist paper at the Italian National Conference on Electromagnetics in 2002 (XIV RiNEm). 